\title{
Neuropathogenesis of severe acute respiratory syndrome coronavirus 2
}

\author{
Payal B. Pate/ ${ }^{\mathrm{a}}$ and David Bearden ${ }^{\mathrm{b}}$
}

\begin{abstract}
Purpose of review
The purpose of this review is to address our current understanding of the pathophysiology of neurologic injury resulting from severe acute respiratory syndrome coronavirus 2 (SARS-CoV2) infection on the developing nervous system.

\section{Recent findings}

SARS-CoV2 may enter the brain through three potential mechanisms: transsynaptic spread from the olfactory bulb following intranasal exposure, migration across the blood-brain barrier through endothelial cell infection, and migration following disruption of the blood-brain barrier from resulting inflammation. SARSCoV2 does not appear to directly infect neurons but rather may produce an inflammatory cascade that results in neuronal injury. Additionally, autoantibodies targeting neuronal tissue resulting from the immune response to SARS-CoV2 are present in select patients and may contribute to central nervous system (CNS) injury.
\end{abstract}

\section{Summary \\ These findings suggest that neuronal injury during SARS-CoV2 infection is immune mediated rather than through direct viral invasion. Further multimodal studies evaluating the pathophysiology of neurologic conditions in pediatric patients specifically following SARS-CoV2 infection are needed to improve our understanding of mechanisms driving neurologic injury and to identify potential treatment options.}

\section{Keywords}

brain, maternal-fetal transmission, neurologic injury, pathophysiology, severe acute respiratory syndrome coronavirus 2

\section{INTRODUCTION}

In late 2019, severe acute respiratory syndrome coronavirus 2 (SARS-CoV2) emerged as pathogen causing a global pandemic of unforeseen proportion. Although pulmonary manifestations of the disease remain the most prominent presentation, neurologic symptoms occur in approximately $30-40 \%$ of patients with acute SARS-CoV2 infection [1]. Recent publications have shed light on the mechanisms by which SARS-CoV2 causes neurologic complications. This review will address our current understanding of the pathophysiology of neurologic invasion and injury resulting from acute SARS-CoV2 infection.

\section{NEUROPATHOLOGIC EVIDENCE OF CENTRAL NERVOUS SYSTEM INJURY FROM PRIOR CORONAVIRUS STRAINS}

Currently, there are seven known coronavirus strains, which have been shown to infect humans. Of these strains, three, severe acute respiratory syndrome coronavirus 1 (SARS-CoV1), Middle East Respiratory Syndrome Coronavirus (MERS-CoV), and SARS-
CoV2, have been associated with severe neurologic disease and both Human Coronavirus OC43 (HCoVOC43) and SARS-CoV1 have demonstrated neuroinvasive potential in murine models $[2,3]$. Acute infection with $\mathrm{HCoV}-\mathrm{OC} 43$ produces productive infection in oligodendrocytes, astrocytes, and neurons in animal models [4]. Similar to one of the proposed mechanisms of spread of SARS-CoV2, $\mathrm{HCoV}-\mathrm{OC} 43$ invades the CNS following intranasal inoculation in animal models [4]. Resultant neuronal injury appears to be virally mediated and clinical presentations include encephalitis and flaccid paralysis in mice. There have been pediatric case reports of $\mathrm{HCoV}-\mathrm{OC} 43$ encephalitis in children with a history

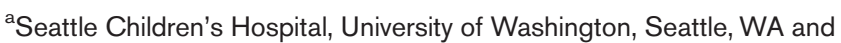
bepartment of Neurology, University of Rochester School of Medicine, Rochester, NY, USA

Correspondence to Payal B. Patel, MD, Acting Assistant Professor, University of Washington, Seattle, USA. Tel: +1 832967 7066;

e-mail: Payal.patel@seattlechildrens.org
}

Curr Opin Pediatr 2021, 33:597-602

DOI:10.1097/MOP.0000000000001068 


\section{KEY POINTS}

- Potential mechanisms of CNS entry of SARS-CoV2 include: transsynaptic spread from the olfactory bulb following intranasal exposure, migration across the blood-brain barrier through endothelial cell infection, and migration following disruption of the blood-brain barrier from resulting inflammation.

- Postmortem analyses and animal models suggest an inflammatory-mediated process for neuronal injury following SARS-CoV2 infection rather than direct viral invasion of neurons.

- Autoantibodies to neuronal tissue have been found in select cases of patients experiencing neurologic symptoms following infection with SARS-CoV2.

- Vertical transmission of SARS-CoV2 infection from mother to child seems to be rare. The long-term neurologic consequences of in-utero SARS-CoV2 exposure remain to determined.

of severe immunosuppression and metagenomic sequencing has confirmed the presence of $\mathrm{HCoV}$ OC43 in cerebral tissue in these cases [5,6]. Additionally, postmortem autopsy studies on individuals living with multiple sclerosis (MS) demonstrate a higher prevalence of HCoV-OC43 RNA in cerebral tissue of individuals living with MS compared with controls without neurologic disease [7]. These data suggest that $\mathrm{HCoV-OC43}$ is neuroinvasive and has the potential to present as a variety of neurologic disorders.

SARS-CoV1 is associated with neurologic disease, as well. Typically SARS-CoV1 infection of the CNS presents as encephalitis. Autopsy studies have demonstrated the presence of SARS-CoV1 in the neuronal cytoplasm of the cortex and hypothalamus [2]. Specimens from patients with encephalitis display necrosis of neurons with surrounding edema and inflammatory infiltration of monocytes and T cells [3]. Intranasal inoculation of SARS-CoV1 in transgenic murine models with human ACE2 expression results in transsynaptic viral spread via the olfactory bulb [8]. This finding is of particular significance given the shared morphology between SARS-CoV1 and SARS-CoV2 including preferential angiotensin-converting enzyme 2 (ACE2) receptors and transmembrane serine protease 2 (TMPRSS2) receptor binding, which are present in the nasal endothelium [9"'].

\section{NEUROINVASIVE POTENTIAL OF SEVERE ACUTE RESPIRATORY SYNDROME CORONAVIRUS 2}

SARS-CoV2 targets ACE2 receptors and transmembrane serine protease 2 (TMPRSS2) receptors on cellular surfaces and within the cytoplasm [9"']. These receptors are expressed on select regions in the central nervous system (CNS) to varying degrees. A study by Chen et al. [10] utilized spatial distribution analyses of publicly available human transcriptome databases in cerebral tissue and identified that ACE2 receptors were highly expressed in cytoplasm of neurons in the prefrontal cortex, hippocampus, posterior cingulate cortex, the middle temporal cortical gyrus, and paraventricular regions of the thalamus, similar to regions of ACE2 receptor expression in murine brains. In contrast, a recent publication by Zhou et al. [11"] and her group utilized a multiomic approach, which included single cell RNA sequencing, proteomics, and interact-onomics (protein-protein interaction) analyses of postmortem cerebral tissue following SARS-CoV2 infection, and demonstrated low levels of expression of ACE2, TMPRS22, and various other SARS-CoV2 entry receptors across multiple brain regions and cellular types. The same group found alteration in genetic expression of inflammatory markers including those associated with cytokine profiling and monocyte translocation suggesting that immunologically mediated injury rather than direct, virally mediated injury is likely to be the cause of neurologic symptoms in patients with SARS-CoV2 [11"].

Potential mechanisms of entry into cerebral tissue have been explored by various studies. These mechanisms include: transsynaptic spread from the olfactory bulb following intranasal exposure, migration across the blood-brain barrier (BBB) through endothelial cell infection, and migration following disruption of the BBB from resulting inflammation. Murine models of mouse hepatitis virus and $\mathrm{HCoV}-\mathrm{OC} 43$ provide support for causal pathway of intranasal inoculation resulting in CNS infection. Pathologic studies have identified a high prevalence of SARS-CoV2 RNA and surface protein structures in olfactory mucosa through quantitative PCR and RNAScope in-situ hybridization techniques [12]. Further evaluation identifying surface proteins of SARS-CoV2 through electron microscopy documented that endothelial cells in these regions were the primary target of infection. SARSCoV2 was not detected in axons in the olfactory bulb, although authors of the study note the limitation of current technology to detect low level viral particles in the small regional area, such as the olfactory nervous system [12]. In the largest autopsy study published to date, Bryce and colleagues found evidence of endothelial dysfunction in multiple organ systems. This dysfunction resulted in a hypercoagulable and hyperinflammatory state with immune-mediated injury noted in neurons surrounding vascular tissue in the brain 
Table 1. Summary of key studies on severe acute respiratory syndrome coronavirus 2 infection neuropathology

\begin{tabular}{|c|c|c|c|}
\hline Reference & $\begin{array}{l}\text { Number } \\
\text { of cases }\end{array}$ & Macroscopic evaluation & Microscopic evaluation \\
\hline Matschke et al. [27] & 43 & Edema, infarcts & $\begin{array}{l}\text { Ischemic infarct; astrogliosis, microgliosis, perivascular } \\
\text { and leptomeningeal T cells }\end{array}$ \\
\hline Solomon et al. [29] & 18 & Atherosclerosis & $\begin{array}{l}\text { Hypoxic/ischemic injury; rare foci of perivascular } \\
\text { lymphocytes and leptomeningeal inflammation }\end{array}$ \\
\hline Hanley [30] & 10 & $\begin{array}{l}\text { Ischemia; } 1 \text { brain with evidence of } \\
\text { brainstem encephalitis }\end{array}$ & $\begin{array}{l}\text { Activation of glial cells, perivascular T-lymphocyte } \\
\text { activation }\end{array}$ \\
\hline $\begin{array}{l}\text { Remmelink et al. } \\
2020[31]\end{array}$ & 17 & Ischemia, hemorrhage, edema & Spongiosis, vascular congestion \\
\hline
\end{tabular}

[13"']. These data suggest that, in select patients, SARS-CoV2 can lead to disruption of vascular function and physiologic barriers potentially resulting in viral spread and inflammation. ACE2 receptors provide a means of communication between endothelial cells and astrocytes and allow for BBB maintenance. Microglia also play an important role in BBB regulation and systemic inflammation may result in disruption of these processes $[14,15]$. Additionally, circumventricular brain regions are surrounded by fenestrated capillaries and may be areas that are susceptible to SARS-CoV2 and inflammatory substrate translocation following systemic infection. These findings have important implications in the developing brain, given the increased permeability of the BBB at younger ages and differential maturation of microglia as individuals age $[16,17]$.

SARS-CoV2 infection may cause neurologic injury through three possible mechanisms: direct infection of neurons resulting in cellular necrosis, immune-mediated injury either through an inflammatory response to the viral infection or the generation of autoantibodies, which target neurons directly, and/or hypoxic/ischemic injury resulting from respiratory compromise or direct injury to the cerebrovascular supply. Pathologic and laboratory evidence for these potential pathways are discussed in detail below.

\section{PATHOLOGIC FEATURES OF NEUROLOGIC INJURY FOLLOWING SEVERE ACUTE RESPIRATORY SYNDROME CORONAVIRUS 2 INFECTION}

Almost all neuropathology studies of SARS-CoV-2 have been performed in adult patients, and thus little is known regarding specific manifestations in children [18]. In addition, the majority of pathology studies have been completed on patients who died after prolonged mechanical ventilation, with many patients having been treated with extracorporeal membrane oxygenation (ECMO). Finally, deaths from COVID-19 and autopsy studies have over-represented older adults and those with preexisting conditions, and thus studies of COVID-19 neuropathology often reflect comorbid disorders common in these populations, such as amyloid deposition and cerebrovascular disease. Thus, it is challenging to sort out specific neuropathologic effects of COVID -19 vs. nonspecific effects of aging, critical illness, and mechanical ventilation [18-21]. Neuropathology studies in adults display diverse manifestations, with the most common being hypoxic/ ischemic changes, reactive gliosis, astrocytosis, and microglial nodules accompanied by neuronophagia $\left[18,22-24,25^{-"}, 26\right]$. In addition, T lymphocytes have been noted to accumulate in perivascular regions [26]. Of note, some studies have identified neuronal damage in the medulla, suggesting that brainstem disorder may play a role in respiratory failure $[27,28]$. A summary of some key neuropathology studies is shown in Table 1.

A single case series of autopsy neuropathology studies of pediatric patients has been published [32]. In this series from Brazil, the most common neuropathology findings were similar to those described above in adults, including reactive microglia and ischemic changes. Of note, a single patient in this series, who had been diagnosed with MIS-C had SARS-CoV-2 identified in the cerebral endothelium and in perivascular astrocytes.

There is little evidence that SARS-CoV-2 typically results in a CNS vasculitis or encephalitis, although there have been rare case reports of this clinical presentation [24,28]. Viral RNA can be detected in select brain tissue, albeit at very low levels $[33,34]$. Viral RNA has been detected in brain tissue by reverse transcription-quantitative realtime PCR but not by in-situ hybridization. This suggests that at least in many cases, the presence of viral RNA may represent contamination by vasculature in leptomeninges and Virchow-Robin spaces [34]. SARS-CoV-2 protein has been found 
in brain vascular endothelium but not typically in neurons or glia on electron microscopy. These findings suggest that the presence of microglial activation, microglial nodules, and neuronophagia observed in the majority of brain tissue does not result from direct viral infection of brain parenchyma but is more likely to be the result of systemic inflammation and hypoxic ischemia [34].

\section{CEREBROSPINAL FLUID ABNORMALITIES IN SEVERE ACUTE RESPIRATORY SYNDROME CORONAVIRUS 2}

Standard cerebrospinal fluid evaluation in most patients with acute SARS-CoV2 infection and neurologic symptoms is usually unremarkable. PCR testing for SARS-CoV2 is often negative except in a few case reports of encephalitis [35]. These cases may represent false positives resulting from blood contamination. Often, there is no clear evidence of inflammation on conventional CSF testing, including a normal white blood cell count and protein in most patients [36"',37]. These data seem to indicate that direct infection with SARS-CoV2 may not be the primary driver of neurologic symptomatology. An alternative explanation is that detection of SARSCoV2 RNA may be limited because of low sensitivity of current PCR testing methods.

Biomarker analyses of cerebrospinal fluid consistently demonstrates abnormal CSF to plasma albumin ratio in a portion of patients with acute SARS-CoV2 infection suggesting breakdown of the BBB during acute infection, particularly in patients experiencing severe SARS-CoV2 infection. Additionally, neurofilament light chain, a marker of neuronal injury, is found to be elevated in the CSF of patients with acute SARS-CoV2 infection and is higher in individuals with severe SARS-CoV2 infection [38]. These data suggest that severe, acute SARSCoV2 infection may result in BBB breakdown and that this process may allow for the extravasation of immune cells or inflammatory cytokines into the CNS space with the potential to cause neuronal injury.

Recent studies evaluating antibody specificity in patients with subacute SARS-CoV2 infection have demonstrated the presence of autoreactive antibodies to neuronal tissue in select patients $\left[39^{-"}, 40^{-1}\right]$. SARS-CoV2 antibodies are able to neutralize pulmonary infection in animal models and also reproducibly bind to cerebral tissue in murine brains suggesting that an autoimmune process may play a role in neurologic injury following SARS-CoV2 infection. The binding pattern of antibodies indicate a predilection for the vessel wall endothelium, perinuclear antigens, astrocytes, and neurons in the basal ganglia and hippocampus, similar to prior reports of binding patterns in other antibody-associated autoimmune encephalitides [39"']. AntiNMDA receptor encephalitis has been reported following SARS-CoV2 infection in case reports including one pediatric patient [41-43]. These findings indicate that autoimmunity may play an important role in the pathophysiology of neurologic injury following SARS-CoV2 infection and a high degree of clinical suspicion for autoimmune encephalitis should be held in patients presenting with hyperexcitable neurologic symptoms, such as seizures or myoclonus, after SARS-CoV2 infection [39"'].

\section{MATERNAL TO FETAL TRANSMISSION OF SEVERE ACUTE RESPIRATORY SYNDROME CORONAVIRUS 2}

The data on the consequences of SARS-CoV-2 infection during pregnancy on both maternal and fetal outcomes is limited. A key concern has been whether SARS-CoV-2 may infect or cross the placenta [44]. A recent review including 1457 pregnancies concluded that the risk of premature birth, maternal death, fetal death, fetal distress, and neonatal asphyxia were all increased in pregnancies affected by SARS-CoV-2 [45]. However, these complications largely seem to result from maternal illness rather than a direct effect of SARS-CoV-2 on the placenta or the fetus $[46,47]$. There is some evidence of histopathologic effects of COVID-19 on the placenta $[48,49]$. In a cohort study of women infected late in pregnancy, SARS-CoV-2 viral RNA was detected in 23 out of 54 placentas [50]. Nevertheless, while vertical transmission of SARS-CoV-2 from mother to child has been described, it appears to be exceedingly rare [51-58]. Long-term neurodevelopmental outcomes of infants who were exposed to SARS-CoV-2 in utero remains an area for future targeted studies [59-61].

\section{CONCLUSION}

In this review, we summarized the current literature regarding the potential mechanism of SARS-CoV2 neuroinvasion and resulting neurologic injury. Although data from pediatric studies are limited, postmortem evaluation of cerebral tissue and CSF evaluation in adults and children and animal model studies suggest an inflammatory mediated process of neuronal injury following SARS-CoV2 infection rather than direct viral invasion of neurons. Further studies are needed in order to determine the pathophysiologic mechanism specific to pediatric patients, particularly in distinct clinical phenotypes, such as multisystem inflammatory syndrome 
in children (MIS-C) and in cohorts of children born to mothers with exposure to SARS-CoV2 infection during pregnancy.

\section{Acknowledgements}

None.

\section{Financial support and sponsorship}

This work was supported by the Department of Neurology, University of Washington, Seattle, Washington, USA and by NIH (NIMH 5K23MH119914).

\section{Conflicts of interest}

P.B.P. has received honoraria from the American Academy of Neurology Continuum and Medlink Neurology as an author and is currently receiving a grant (5K23MH119914) from the National Institute of Health. D.B. is supported by the National Institute of Neurological Disorders and Stroke of the National Institutes of Health under Award Number K23NS117310. The content is solely the responsibility of the authors and does not necessarily represent the official views of the National Institutes of Health. D.B. has served as a paid consultant for Biogen and Praxis Precision Medicines.

\section{REFERENCES AND RECOMMENDED READING}

Papers of particular interest, published within the annual period of review, have been highlighted as:

- of special interest

I. of outstanding interest

1. Ellul MA, Benjamin L, Singh B, et al. Neurological associations of COVID-19. Lancet Neurol 2020; 19:767-783.

2. Ding $Y$, Wang $H$, Shen $H$, et al. The clinical pathology of severe acute respiratory syndrome (SARS): a report from China. J Pathol 2003; 200:282-289.

3. Gu J, Gong E, Zhang B, et al. Multiple organ infection and the pathogenesis of SARS. J Exp Med 2005; 202:415-424.

4. Jacomy $\mathrm{H}$, Talbot $\mathrm{P}$. Vacuolating encephalitis in mice infected by human coronavirus OC43. Virology 2003; 315:20-33.

5. Nilsson A, Edner N, Albert J, Ternhag A. Fatal encephalitis associated with coronavirus OC43 in an immunocompromised child. Infect Dis (Lond) 2020; $52: 419-422$.

6. Morfopoulou S, Brown JR, Davies EG, et al. Human coronavirus OC43 associated with fatal encephalitis. N Engl J Med 2016; 375:497-498.

7. Arbour N, Day R, Newcombe J, Tablot P. Neuroinvasion by human respiratory coronaviruses. J Virol 2000; 74:8913-8921.

8. Netland J, Meyerholz DK, Moore S, et al. Severe acute respiratory syndrome coronavirus infection causes neuronal death in the absence of encephalitis in mice transgenic for human ACE2. J Virol 2008; 82:7264-7275.

9. Hoffmann M, Kleine-Weber H, Schroeder S, et al. SARS-CoV-2 cell entry

- depends on ACE2 and TMPRSS2 and is blocked by a clinically proven protease inhibitor. Cell 2020; 181:271.e8-280.e8.

Authors demonstrate that SARS-CoV-2 uses the ACE2 receptor for entry and the serine protease TMPRSS2 receptor for $S$ protein priming.

10. Chen R, Wang K, Yu J, et al. The spatial and cell-type distribution of SARSCoV-2 receptor ACE2 in the human and mouse brains. Front Neurol 2021; 11:573095.

11. Zhou $Y, X u J$, Hou $Y$, et al. Network medicine links SARS-CoV-2/COVID-19

- infection to brain microvascular injury and neuroinflammation in dementia-like cognitive impairment. Alz Res Therapy 2021; 13:110.

Mechanism of neurologic injury following SARS-CoV2 infection include inflammation and vascular insult.

12. Meinhardt J, Radke J, Dittmayer $C$, et al. Olfactory transmucosal SARS-CoV-2 invasion as a port of central nervous system entry in individuals with COVID19. Nat Neurosci $2021 ; 24: 168-175$.
13. Bryce C, Grimes Z, Pujadas E, et al. Pathophysiology of SARS-CoV-2: the - Mount Sinai COVID-19 autopsy experience. Mod Pathol 2021; 34:1456-1467.

Pathology studies following SARS-CoV2 infection demonstrate microthrombi in multiple organ systems including the brain. This is thought to result in a hypercoagulable and proinflammatory state with the most prominent feature being endothelial dysfunction.

14. Wosik K, Cayrol R, Dodelet-Devillers A, et al. Angiotensin II controls occludin function and is required for blood brain barrier maintenance: relevance to multiple sclerosis. J Neurosci 2007; 27:9032-9042.

15. Thurgur H, Pinteaux E. Microglia in the neurovascular unit: blood-brain barrier microglia interactions after central nervous system disorders. Neuroscience 2019; 405:55-67.

16. Erdo F, Denes $L$, de Lange $E$. Age-associated physiological and pathological changes at the blood-brain barrier: a review. J Cereb Blood Flow Metab 2017; $37: 4-24$.

17. Ek CJ, Dziegielewska KM, Habgood MD, et al. Barriers in the developing brain and neurotoxicology. Neurotoxicology 2012; 33:586-604.

18. Fisicaro F, Di Napoli $M$, Liberto $A$, et al. Neurological sequelae in patients with COVID-19: a histopathological perspective. Int J Environ Res Public Health $2021 ; 18: 1415$.

19. Glatzel M, Hagel $C$, Matschke J, et al. Neuropathology associated with SARSCoV-2 infection. Lancet 2021; 397:276.

20. Egervari K, Thomas C, Lobrinus JA, et al. Neuropathology associated with SARS-CoV-2 infection. Lancet 2021; 397:276-277.

21. Deigendesch $N$, Sironi $L$, Kutza M, et al. Correlates of critical illness-related encephalopathy predominate postmortem COVID-19 neuropathology. Acta Neuropathol 2020; 140:583-586.

22. Morgello S. Coronaviruses and the central nervous system. J Neurovirol 2020; 26:459-473.

23. Pajo AT, Espiritu AI, Apor ADAO, Jamora RDG. Neuropathologic findings of patients with COVID-19: a systematic review. Neurol Sci 2021; 42:1255-1266

24. Caramaschi S, Kapp ME, Miller SE, et al. Histopathological findings and clinicopathologic correlation in COVID-19: a systematic review. Mod Pathol $2021 ; 34: 1614-1633$.

25. Thakur KT, Miller EH, Glendinning MD, et al. COVID-19 neuropathology at

- Columbia University Irving Medical Center/New York Presbyterian Hospital. Brain 2021; awab148. doi: 10.1093/brain/awab148. [Epub ahead of print] This case series of pathologic cerebral samples strongly suggests that microglial activation, microglial nodules and neuronophagia, observed in the majority of brains, do not result from direct viral infection of brain parenchyma but from systemic inflammation and/or hypoxia.

26. Yachou Y, El Idrissi A, Belapasov V, Ait Benali S. Neuroinvasion, neurotropic, and neuroinflammatory events of SARS-CoV-2: understanding the neurological manifestations in COVID-19 patients. Neurol Sci 2020; 41:2657-2669.

27. Matschke J, Lütgehetmann M, Hagel C, et al. Neuropathology of patients with COVID-19 in Germany: a postmortem case series. Lancet Neurol 2020; 19:919-929.

28. Bulfamante G, Bocci $T$, Falleni $M$, et al. Brainstem neuropathology in two cases of COVID-19: SARS-CoV-2 trafficking between brain and lung. J Neurol 2021; 1-6. doi: 10.1007/s00415-021-10604-8. [Epub ahead of print]

29. Solomon $\mathrm{IH}$, Normandin $\mathrm{E}$, Bhattacharyya $\mathrm{S}$, et al. Neuropathological features of Covid-19. N Engl J Med 2020; 383:989-992.

30. Hanley B, Naresh KN, Roufosse C, et al. Histopathological findings and viral tropism in UK patients with severe fatal COVID-19: a postmortem study. Lancet Microbe 2020; $1:$ e245-e253.

31. Remmelink M, De Mendonça R, D'Haene N, et al. Unspecific postmortem findings despite multiorgan viral spread in COVID-19 patients. Crit Care 2020; 24:495

32. Duarte-Neto AN, Caldini EG, Gomes-Gouvêa MS, et al. An autopsy study of the spectrum of severe COVID-19 in children: from SARS to different phenotypes of MIS-C. EClinicalMedicine 2021; 35:100850.

33. Bodro M, Compta $Y$, Sánchez-Valle R. Presentations and mechanisms of CNS disorders related to COVID-19. Neurol Neuroimmunol Neuroinflamm 2021; 8:e923.

34. Boldrini M, Canoll PD, Klein RS. How COVID-19 affects the brain. JAMA Psychiatry 2021; 78:682-683.

35. Espindola OM, Siqueira M, Soares CN, et al. Patients with COVID-19 and neurological manifestations show undetectable SARS-CoV-2 RNA levels inthe cerebrospinal fluid. Int J Infect Dis 2020; 96:567-569.

36. Neumann B, Schmidbauer ML, Dimitriadis K, et al., PANDEMIC and the

- IGNITE study groups. Cerebrospinal fluid findings in COVID-19 patients with neurological symptoms. J Neurol Sci 2020; 418:117090.

Large (30 patient) cohort of patients with acute COVID-19 who underwent LP; none had SARS-CoV-2 detectable in the CSF.

37. Bellon $M$, Schweblin $C$, Lambeng $N$, et al. Cerebrospinal fluid features in SARS-CoV-2 RT-PCR positive patients. Clin Infect Dis 2020; ciaa1165. doi: 10.1093/cid/ciaa1165. [Epub ahead of print]

38. Garcia MA, Barreras PV, Lewis A, et al. Cerebrospinal fluid in COVID-19 neurological complications: neuroaxonal damage, anti-SARS-Cov2 antibodies but no evidence of cytokine storm. J Neurol Sci 2021; 427:117517. 
39. Franke C, Ferse C, Kreye J, et al. High frequency of cerebrospinal fluid -1. autoantibodies in COVID-19 patients with neurological symptoms. Brain Behav Immun 2021; 93:415-419.

Blood and CSF samples from 11 patients with severe COVID-19 illness showed autoantibodies to murine model cerebral tissue, including anti-Yo and anti-NMDA receptor antibodies. A high frequency of autoantibodies to neuronal tissue may be seen in patients with SARS-CoV2 infection and clinical features of hyperexcitable syndromes, such as seizures.

40. Song $E$, Bartley $C M$, Chow RD, et al. Divergent and self-reactive immune

- responses in the CNS of COVID-19 patients with neurological symptoms. Cell Rep Med 2021; 2:100288.

Compartmentalized immunologic response occur in the CNS following SARSCoV2 infection in select patient including monoclonal antibodies that target antineural antigens.

41. Monti G, Giovannini G, Marudi A, et al. Anti-NMDA receptor encephalitis presenting as new onset refractory status epilepticus in COVID-19. Seizure 2020; 81:18-20.

42. Panariello $A$, Bassetti $R$, Radice $A$, et al. Anti-NMDA receptor encephalitis in a psychiatric Covid-19 patient: a case report. Brain Behav Immun 2020; 87:179-181.

43. Burr $T$, Barton $C$, Doll E, et al. N-Methyl-d-aspartate receptor encephalitis associated with COVID-19 infection in a toddler. Pediatr Neurol 2021; 114:75-76.

44. Aghaamoo S, Ghods K, Rahmanian M. Pregnant women with COVID-19: the placental involvement and consequences. J Mol Histol 2021; 52:427-435.

45. Amaral WND, Moraes CL, Rodrigues APDS, et al. Maternal coronavirus infections and neonates born to mothers with SARS-CoV-2: a systematic review. Healthcare (Basel) 2020; 8:E511.

46. Kumar R, Yeni CM, Utami NA, et al. SARS-CoV-2 infection during pregnancy and pregnancy-related conditions: concerns, challenges, management and mitigation strategies-a narrative review. J Infect Public Health 2021; 14:863-875.

47. Jafari M, Pormohammad A, Sheikh Neshin SA, et al. Clinical characteristics and outcomes of pregnant women with COVID-19 and comparison with control patients: a systematic review and meta-analysis. Rev Med Virol 2021; $31: 1-16$.

48. Hosier H, Farhadian SF, Morotti RA, et al. SARS-CoV-2 infection of the placenta. J Clin Invest 2020; 130:4947-4953.
49. Resta L, Vimercati A, Cazzato G, et al. SARS-CoV-2 and placenta: new insights and perspectives. Viruses 2021; 13:723.

50. Argueta LB, Lacko LA, Bram $Y$, et al. SARS-CoV-2 infects syncytiotrophoblast and activates inflammatory responses in the placenta. bioRxiv 2021. doi: 10.1101/2021.06.01.446676.

51. Mirbeyk M, Saghazadeh A, Rezaei N. A systematic review of pregnant women with COVID-19 and their neonates. Arch Gynecol Obstet 2021; 304:5-38.

52. Moore KM, Suthar MS. Comprehensive analysis of COVID-19 during pregnancy. Biochem Biophys Res Commun 2021; 538:180-186.

53. Oliveira KF, Oliveira JF, Wernet $M$, et al. Vertical transmission and COVID-19: a scoping review. Rev Bras Enferm 2021; 74:e20200849.

54. Palalioglu RM, Mahammadaliyeva A, Erbiyik HI, Muhcu M. COVID-19 in third trimester may not be as scary as you think, it can be innocent: evaluating vertical transmission from a COVID-19 positive asymptomatic pregnant woman with early membrane rupture. J Obstet Gynaecol Res 2021; 47: 838-842.

55. Dube R, Kar SS. COVID-19 in pregnancy: the foetal perspective-a systematic review. BMJ Paediatr Open 2020; 4:e000859.

56. Karimi-Zarchi M, Neamatzadeh $H$, Dastgheib SA, et al. Vertical transmission of coronavirus disease 19 (COVID-19) from infected pregnant mothers to neonates: a review. Fetal Pediatr Pathol 2020; 39:246-250.

57. Garcia-Ruiz I, Sulleiro E, Serrano B, et al., GESTACOVID Collaborative Group. Congenital infection of SARS-CoV-2 in live-born neonates: a population-based descriptive study. Clin Microbiol Infect 2021; 27:1521.e1-1521.e5. doi: 10.1016/j.cmi.2021.06.016. [Epub ahead of print]

58. He JR, Xiao YH, Ding W, et al. Maternal, placental and neonatal outcomes after asymptomatic SARS-CoV-2 infection in the first trimester of pregnancy: a case report. Case Rep Womens Health 2021; 31:e00321.

59. Hoffman MC, Freedman R, Law AJ, et al. Maternal nutrients and effects of gestational COVID-19 infection on fetal brain development. Clin Nutr ESPEN $2021 ; 43: 1-8$

60. Trippella G, Ciarcià M, Ferrari M, et al. COVID-19 in pregnant women and neonates: a systematic review of the literature with quality assessment of the studies. Pathogens 2020; 9:E485.

61. Yoon SH, Kang JM, Ahn JG. Clinical outcomes of 201 neonates born to mothers with COVID-19: a systematic review. Eur Rev Med Pharmacol Sci $2020 ; 24: 7804-7815$ 